\title{
3 Research Suare \\ Procalcitonin levels in children with bloodstream infections caused by different species: a cohort study
}

\section{José Iván Castillo Bejarano}

University Hospital "Dr. José Eleuterio González" https://orcid.org/0000-0003-4270-2687

\section{Agustín De Colsa Ranero}

Hospital Universitario Dr Jose Eleuterio Gonzalez

Oscar Tamez Rivera

Instituto Nacional de Pediatria

Andrés Guillen Lozoya

Hospital Universitario Dr Jose Eleuterio Gonzalez

Napoleón González Saldaña

Instituto Nacional de Pediatria

Alfonso Huante Anaya

Instituto Nacional de Pediatria

Ismael Herrera Benavente

Universidad Autonoma de San Luis Potosi - Facultad de Medicina

Abiel Mascareñas de los Santos ( $\nabla$ a_mascarenas@hotmail.com )

https://orcid.org/0000-0002-4057-9526

Research article

Keywords: Procalcitonin; Sepsis; Bacterial sepsis

Posted Date: April 30th, 2020

DOI: https://doi.org/10.21203/rs.3.rs-25485/v1

License: (1) (1) This work is licensed under a Creative Commons Attribution 4.0 International License.

Read Full License 


\section{Abstract}

Background: Timely diagnosis and accurate identification of the causative microorganism in sepsis is crucial in order to offer targeted treatment and increase survival rates. Previous studies have aimed to identify biomarkers that could potentially predict blood culture positivity in patients with bacteremia; however, most of the research has been performed in adult populations. The aims of this study were to analyze procalcitonin (PCT) levels in confirmed bloodstream infections by species in children and assess their utility in immunocompromised patients.

Methods: Medical records of children younger than 18 years admitted from 2011 to 2018 were reviewed. Subjects who met the diagnostic criteria for sepsis, with PCT levels collected within a 72-hour period prior to obtaining a blood culture were included. Kruskal-Wallis test was used to compare differences among groups. Receiver-operating characteristic curves were used to evaluate PCT cut-offs.

Results: A total of 120 patients were included. Mean age was 55 months. Mean PCT levels in immunosuppressed patients was $26.68 \mathrm{mcg} / \mathrm{L}$, compared to 8.78 in the immunocompetent group. Subjects with bacteremia by Gram-negative bacilli (GNB) had the highest mean PCT levels (18.2 \pm 34.2$)$ ( $p<0.001$ ). Sensitivity and specificity were $78 \%$ and $53 \%$ for Gram-positive cocci (GPC), $60.9 \%$ and $33.3 \%$ for GNB, and $75 \%$ and $25 \%$ for yeasts, respectively. Subgroup analysis showed $87.5 \%$ sensitivity and $16.7 \%$ specificity of PCT for predicting documented GNB bacteremia in immunodeficient children.

Conclusions: PCT may be considered as a surrogate biomarker in immunocompromised children, and a viable tool to differentiate etiology by species.

\section{Background}

Sepsis is a leading cause of morbidity and mortality in the pediatric population (1). Timely diagnosis and accurate identification of the causative microorganism is crucial in order to offer targeted treatment and increase survival rates. Previous studies have aimed to identify biomarkers that could potentially predict blood culture positivity in patients with bacteremia; however, most of the research has been performed in adult populations $(1,2)$.

Procalcitonin (PCT) is a 116-aminoacid prohormone synthetized and secreted by the thyroid C-cells (35). High levels of PCT are observed in critically ill infected patients (7-10). It has been reported that PCT levels higher than $3.61 \mathrm{ng} / \mathrm{mL}$ predict blood culture-confirmed bacteremia in adults, with a $75 \%$ sensitivity and $72 \%$ specificity (9). Likewise, previous studies have described a high negative predictive value (95.4\%) of normal levels of PCT for predicting bacteremia (10). Furthermore, it has been suggested that PCT levels could be helpful in differentiating among bacterial species in adults, with Gram-negative bacilli (GNB) causing a greater increase of PCT levels compared to Gram-positive cocci (GPC) (11). The objectives of this study were to analyze PCT levels in confirmed bloodstream infections by species in children and assess their utility in immunocompromised patients. 


\section{Methods}

Medical records of all subjects admitted to the National Institute of Pediatrics (INP) in Mexico City from 2011 to 2018 were reviewed. Subjects younger than 18 years of age who met the diagnostic criteria for sepsis, with PCT levels collected within a 72-hour period prior to obtaining a blood culture were included in the analysis. Subjects with polymicrobial blood cultures and/or with isolation of commensal bacteria (coagulase-negative Staphylococci, Gram-positive bacilli, and Micrococcus spp) in a single peripheral blood culture were excluded. Blood cultures with commensal bacteria were only included in central lineassociated bloodstream infections (CLABSI), and if the same microorganism was documented in two or more peripheral blood cultures. Samples were processed by the automated blood culture system BD BACTEC $^{\text {TM }}$. Bacterial identification and susceptibility testing were performed by BD Phoenix ${ }^{\text {TM }} 100$. PCT levels were obtained using the Thermo Scientific Fisher ${ }^{\mathrm{TM}}$ system, with a cut-off value of $0.5 \mathrm{mcg} / \mathrm{L}$.

\subsection{Definitions}

Sepsis was defined as the presence of systemic inflammatory response syndrome (SIRS) in addition to a documented infection via blood culture. SIRS was defined according to the Society of Critical Care Medicine (4). Subjects who met two or more of the following criteria were diagnosed with SIRS: fever $\geq$ $38^{\circ} \mathrm{C}$, hypothermia $<36^{\circ} \mathrm{C}$, tachycardia $>90$ beats per minute, tachypnea $>20$ breaths per minute, hypocapnia $\mathrm{PaCO}_{2}<32 \mathrm{mmHg}$, and leukocytosis/leukopenia adjusted by age. In order to avoid overestimation of infectious episodes, a second infectious event in the same subject was defined as sepsis after a minimum of six days of hemodynamic stability without antibiotics. Patients with immunodeficiencies, including solid organ transplantation, hematopoietic stem cell transplantation (HSCT), primary immunodeficiency, solid tumors, nephrotic syndrome, Down's syndrome, severe malnutrition, hematologic immunodeficiency (i.e. leukemia, hemophagocytic lymphohistiocytosis), and drug-induced immunodeficiency were eligible for inclusion in the study.

\subsection{Statistical analysis}

For the statistical analysis, chi-squared test was used to analyze categorical variables. Kruskal-Wallis test was used to compare differences among groups. Receiver-operating characteristic (ROC) curves were used to evaluate PCT cut-offs. Statistical significance was assumed if the null hypothesis could be rejected at $p<0.05$. Statistical analysis was performed using SPSS v.21 (IBM Corp., USA).

\section{Results}

\subsection{Demographic characteristics}

A total of 41,836 blood cultures were obtained from January 2011 to April 2018, of which 5,059 cultures (12.09\%) were positive. PCT levels were available for 311 subjects; 191 of these were excluded due to polymicrobial $(n=2)$, contaminated $(n=68)$, and duplicated $(n=121)$ blood cultures. The final sample included 120 subjects with sepsis and documented PCT levels within a 72-hour period before blood 
culture collection (Fig. 1). Mean age was 55 months, $54 \%$ were male, and $44.2 \%$ had an immunodeficiency. Mean PCT level was $15.3 \mathrm{mcg} / \mathrm{L}$.

\subsection{PCT levels in immunocompromised children}

Mean PCT level in immunodeficient children was $26.68 \mathrm{mcg} / \mathrm{L}$, compared to $8.78 \mathrm{mcg} / \mathrm{L}$ in immunocompetent hosts $(p<0.05)$. PCT level distribution is shown in Table 1 . The most frequent immunodeficiencies were hematologic (53.8\%), primary immunodeficiency $(9.6 \%)$, and solid tumors (9.6\%). Subjects with hematologic immunodeficiency had the highest PCT mean level $(31.4 \mathrm{mcg} / \mathrm{L})$, followed by HSCT recipients ( $16.9 \mathrm{mcg} / \mathrm{L})$; however, no statistical significance was observed.

Table 1

PCT levels by type of immunodeficiency

\begin{tabular}{|c|c|c|c|}
\hline Type of ID ${ }^{1,2}$ & $\mathrm{n}(\%)$ & Median PCT level (mcg/L) & $P$ value \\
\hline SOT $^{2}$ & $2(3.8)$ & 2.4 & 0.15 \\
\hline PID $^{2}$ & $5(9.6)$ & 3.8 & \\
\hline Hematology & $28(53.8)$ & 31.4 & \\
\hline $\mathrm{HSCT}^{2}$ & $2(3.8)$ & 23.8 & \\
\hline Solid tumor & $5(9.6)$ & 9.3 & \\
\hline Nephrotic syndrome & $3(5.8)$ & 16.9 & \\
\hline Down syndrome & $3(5.8)$ & 1.14 & \\
\hline Severe malnutrition & $3(5.8)$ & 1.9 & \\
\hline Drugs & $1(1.9)$ & 180 & \\
\hline \multicolumn{4}{|c|}{ 1) Kruskal-Wallis analysis } \\
\hline
\end{tabular}

\subsection{PCT levels by microorganism}

Mean PCT levels by microorganism are shown in Table 2 . The most commonly isolated microorganisms were GNB (65.8\%), followed by GPC (24.2\%) and yeasts (10\%). Subjects with Gram-negative bacterial sepsis had the highest mean PCT levels $(18.2 \pm 34.2 \mathrm{mcg} / \mathrm{L})$, compared to GPC $(13.1 \pm 36 \mathrm{mcg} / \mathrm{L})$ and yeasts $(1.9 \pm 1.69 \mathrm{mcg} / \mathrm{L})$. We found a statistically significant difference in mean PCT levels among the major microorganism groups $(\mathrm{p}<0.001)$. The bacteria with highest PCT mean levels were Beta-hemolytic streptococcus (39.3 mcg/L), Klebsiella pneumoniae (28.4 mcg/L), and Streptococcus pneumoniae (25.8 $\mathrm{mcg} / \mathrm{L})$. 
Table 2

PCT levels corresponding to pathogens isolated

\begin{tabular}{|c|c|c|c|}
\hline Pathogen & n (\%) & Median PCT level (mcg/L) & Range \\
\hline Gram-negative bacteria & 79 & 18.2 & $0.3-243.4$ \\
\hline Acinetobacter spp & $3(2.5)$ & 3.4 & $0.99-8.16$ \\
\hline Burkholderia cepacia & $2(1.7)$ & 0.1 & $0.09-0.21$ \\
\hline Enterobacter cloacae & $7(5.8)$ & 14.2 & $0.18-45.8$ \\
\hline E. coli & $18(15)$ & 16.9 & $0.26-93.6$ \\
\hline Klebsiella spp & $25(20.8)$ & 28.4 & $0.46-243.4$ \\
\hline Salmonella spp & $3(2.5)$ & 13.7 & $0.43-34.9$ \\
\hline Stenotrophomonas maltophiliia & $6(5)$ & 7.9 & $0.03-40$ \\
\hline Pseudomonas aeruginosa & $15(12.5)$ & 15.1 & $0.33-76.6$ \\
\hline Gram-positive bacteria & 29 & 13.1 & $0.03-180$ \\
\hline Enterococcus spp & $3(2.5)$ & 1 & $0.4-2.2$ \\
\hline Staphylococcus aureus & $8(6.7)$ & 0.8 & $0.7-3$ \\
\hline Coagulase negative staphylococcus & $11(9.2)$ & 17.3 & $0.1-180$ \\
\hline Staphylococcus lugdunensis & $2(1.7)$ & 5.1 & $0.03-101$ \\
\hline Beta-hemolytic streptococcus & $3(2.5)$ & 39.3 & $7.3-65$ \\
\hline Streptococcus pneumoniae & $2(1.7)$ & 25.8 & $1.2-50.4$ \\
\hline Fungi & 12 & 1.9 & $0.2-5.5$ \\
\hline Candida spp & $12(10)$ & 1.9 & $0.2-5.5$ \\
\hline
\end{tabular}

\subsection{PCT sensitivity and specificity by microorganism}

ROC curves were used to evaluate the diagnostic efficacy of PCT for predicting a positive blood culture. Using a cut-off value of $0.5 \mathrm{mcg} / \mathrm{L}$, we found a sensitivity of $58 \%$ and specificity of $35 \%$, with an area under the curve (AUC) of $0.639(95 \% \mathrm{Cl}, 0.519-0.760)$. Higher values were observed in immunocompromised subjects with $82 \%$ sensitivity and $53 \%$ specificity (AUC $0.635,95 \%$ Cl. $0.457-$ 0.812 ). Sensitivity and specificity by microorganism group were $78 \%$ and $53 \%$ for GPC, respectively (AUC $0.581,95 \% \mathrm{Cl}, 0.402-0.761$ ), compared to $60.9 \%$ and $33.3 \%$ for GNB (AUC $0.640,95 \% \mathrm{Cl}, 0.429-0.851$ ). For yeasts, we found a $75 \%$ sensitivity and $25 \%$ specificity (AUC $0.734,95 \% \mathrm{Cl}, 0.444-1$ ).

Subgroup analysis showed an $87.5 \%$ sensitivity and $16.7 \%$ specificity of PCT for predicting blood culturedemonstrated GNB infection in immunocompromised patients (AUC 0.906, 95\% Cl, 0.748-1), and a 
$78.8 \%$ sensitivity and $22.2 \%$ specificity for GPC infection (AUC $0.744,95 \% \mathrm{Cl}, 0.542-0.946$ ).

\section{Discussion}

The current knowledge about PCT as a biomarker for sepsis in children has been described in previous studies (12-13). Elevation of PCT levels usually occurs earlier during the course of infection, even before the elevation of other biomarkers, peaking at 24-36 hours (12). Pontrelli et al (13) showed a moderate accuracy for the diagnosis of sepsis in neonates with a PCT cut-off of 2.0-2.5 ng/mL (14). A 2015 metaanalysis showed that PCT is highly accurate in differentiating bacterial and viral meningitis in children with $96 \%$ sensitivity (12). PCT leves in blood culture positivity by different microorganism groups in children is scarce, especially in immunocompromised hosts.

Our results show a statistically significant difference between mean PCT values for each microorganism group. Mean PCT levels in children with GNB infections were significantly higher than those with GPC and fungal infections. These findings are consistent with previous studies performed in adults (14-16). Yan et al (1) reported a $72.4 \%$ sensitivity and $51 \%$ specificity of PCT as a predictor of blood culture positivity in adults, using a $0.495 \mathrm{mcg} / \mathrm{L}$ PCT cut-off value. Watanabe et al (17), reported a $74.5 \%$ sensitivity and $59.1 \%$ specificity of PCT for predicting blood culture-proven bacteremia. In our study we found a $75 \%$ sensitivity and $53 \%$ specificity of PCT as a predictor of GNB infection, using a PCT cut-off value of 0.5 $\mathrm{mcg} / \mathrm{L}$.

Thomas-Rüddel et al (18) reported a median PCT significantly higher in GNB compared to GPC $(26 \mathrm{ng} / \mathrm{ml}$ vs $7.1 \mathrm{ng} / \mathrm{ml}, \mathrm{p}<0.001)$. The AUC in the ROC analysis was 0.69 (0.67-0.72) for differentiating GNB from GPC or candidemia, and $0.73(0.71-0.74)$ for the prediction of GNB compared to all other blood culture results. Bassetti $M$ et al (19), reported similar findings with a median PCT concentration of $25.1 \mathrm{ng} / \mathrm{ml}$ in GNB bacteremia compared to $8.9 \mathrm{ng} / \mathrm{ml}$ in GPC. The AUC was $0.7(0.62-0.77)$ among GNB and 0.46 (0.39-0.53) among GPB. In a previous study, Shuhua et al (20) found a median PCT level of $7.47 \mathrm{ng} / \mathrm{ml}$ in GNB compared to $0.48 \mathrm{ng} / \mathrm{ml}$ in GPC. An optimal cut-off value of $3.11 \mathrm{ng} / \mathrm{mL}$ for PCT in discriminating GNB sepsis from fungal sepsis, led to a sensitivity of $63.9 \%$ and specificity of $93.3 \%$.

The role of PCT as a predictor of GPC in blood cultures, mainly in infections caused by staphylococci was evaluated by Shomali et al, reporting higher mean PCT levels in infections by $S$. aureus compared to coagulase-negative staphylococci $(0.85 \mathrm{mcg} / \mathrm{L}$ versus $0.26 \mathrm{mcg} / \mathrm{L}$, respectively) (21). In our study we found higher PCT levels in bloodstream infections by coagulase-negative staphylococci (17.3 vs $0.8 \mathrm{ng} / \mathrm{mL}$ ). This difference may be attributed to a higher isolation rate of coagulase-negative staphylococci in our hospital.

Studies that analyze PCT as a biomarker for invasive fungal infection by Candida spp are scarce and show conflicting data (22-25). In our study, mean PCT levels in Candida spp infections were $1.9 \mathrm{mcg} / \mathrm{L}$, with a $75 \%$ sensitivity and $25 \%$ specificity. Previous studies by Cortegiani et al (25) report higher sensitivity and specificity of PCT for predicting fungal infection by Candida spp, with $86.8 \%$ and $87.4 \%$ respectively. Identification of Candida species was not performed in our study; however, previous authors 
have not found any difference regarding PCT levels in infections by different Candida species. ThomasRüddel et al (18) reported a median PCT level of $4.7 \mathrm{ng} / \mathrm{ml}$, compared to $2.1 \mathrm{ng} / \mathrm{ml}$ by Bassetti et al (19). Median PCT levels of $0.6 \mathrm{ng} / \mathrm{ml}, 0.5 \mathrm{ng} / \mathrm{ml}, 1 \mathrm{ng} / \mathrm{ml}$ and $0.5 \mathrm{ng} / \mathrm{ml}$ were reported by Shuhua et al (20), Miglietta et al (26), Oussalah et al (27) and Leli et al (29), respectively. Consistent with previous studies, we report lower PCT levels in fungal infections compared to bacterial events (26). It has been suggested that fungal infections could trigger an alternate inflammatory response route that does not involve PCT, explaining its modest rise.

Studies on PCT in immunocompromised patients are scarce (30-34). A recent systematic review and meta-analysis in children with chemotherapy-induced neutropenic fever showed that PCT levels > $0.5 \mathrm{ng} / \mathrm{mL}$ have a $67 \%$ sensitivity $(\mathrm{Cl} 0.53-0.79)$, and $73 \%$ specificity $(\mathrm{Cl} 0.66-0.77)$ for predicting microbiologically defined infections (34). In our study according to mean PCT levels, we found a statistically significant difference between immunocompromised $(26.68 \mathrm{mcg} / \mathrm{L})$ and immunocompetent $(8.78 \mathrm{mcg} / \mathrm{L})$ children with sepsis $(p<0.05)$. We also report an $87.5 \%$ sensitivity of PCT for predicting blood culture-proven GNB infection, making PCT a useful resource in clinical practice. PCT levels were also increased in different types of immunosuppression.

Our study has several limitations. A prospective design would aid in having better control of the variables and include a larger sample. Likewise, PCT measurements were not serial, which would have allowed us to analyze PCT behavior in relation to variables such as time, isolated microorganism, treatment, and outcome.

\section{Conclusions}

Our study found that PCT could be a viable tool to predict blood-culture proven sepsis, particularly in immunocompromised patients with GNB infection. The use of PCT could be considered as a surrogate biomarker of bacterial infection, and PCT levels could offer a general prediction of the possible microbial etiology (GNB, GPC and yeasts). Further prospective studies are needed in order to expand the available evidence on the use of PCT as a predictive value for blood culture-proven by species infection in children.

\section{List Of Abbreviations}

PCT - Procalcitonin

GNB - Gram-negative bacilli

GPC - Gram-positive cocci

CLABSI - Central line-associated bloodstream infections

SIRS - Systemic inflammatory response syndrome

HSCT - Hematopoietic stem cell transplantation 
ROC - Receiver-operating characteristic

AUC - Area under the curve

\section{Declarations}

Ethics approval and consent to participate: The authors confirm our study was submitted to and approved by the Academic Group of the National Institute of Pediatrics, Mexico, with the code:

GA/094/18. No informed consent was obtained due to the nature of the retrospective data according to the Academic Group of the National Institute of Pediatrics, Mexico. The data used in this study was anonymized before its use.

Consent for publication: Not applicable

Availability of data and materials: Data sharing is not applicable to this article as no datasets were generated or analyzed during the current study.

Competing interests: The authors declare that they have no competing interests

Funding: This research did not receive any specific grant from funding agencies in the public, commercial, or not-for-profit sectors.

Authors' contributions: CBJI conception and analysis of data; CRA interpretation of the data, TRO analysis and writing, GLA statistical analysis, GSN design of the work, HAA creation of a software used in the work, HBI have substantively revised the work, MSAH work coordination and analysis.

Authors' information: Not applicable

Acknowledgements: Not applicable

\section{References}

1. Yan ST, Sun LC, Jia HB, Gao W, Yang JP, Zhang GQ. Procalcitonin levels in bloodstream infections caused by different sources and species of bacteria. Am J Emerg Med. 2017;35(4):579-83.

2. Liu HH, Zhang MW, Guo JB, Li J, Su L. Procalcitonin and C-reactive protein in early diagnosis of sepsis caused by either Gram-negative or Gram-positive bacteria. Ir J Med Sci. 2017;186(1):207-12.

3. Fran Balamuth, Scott L. Weiss, Mark I. Neuman, Halden Scott, Patrick W. Brady, Raina Paul, Reid W.D. Farris, Richard McClead, Katie Hayes, David Gaieski, Matt Hall S, S. Shah; Elizabeth R. Alpern. Pediatric Severe Sepsis in US Children's Hospitals. Pediatr Crit Care Med. 2014;15(9):798-805.

4. Singer, M. et al., Bellomo R, Bernard GR, Chiche J, Craig M, Hotchkiss RS, et al. The Third International Consensus Definitions for Sepsis and Septic Shock (Sepsis-3). JAMA. 2016;315(8):801-10. 
5. Hawiger J, Veach RA, Zienkiewicz J. New paradigms in sepsis: From prevention to protection of failing microcirculation. J Thromb Haemost. 2015;13(10):1743-56

6. Simon L, Gauvin F, Amre DK, Saint-Louis P, Lacroix J. Serum procalcitonin and C-reactive protein levels as markers of bacterial infection: A systematic review and meta-analysis. Clin Infect Dis. 2004;39(2):206-17.

7. Casado Flores J, Blanco Quirós Procalcitonina: un nuevo marcador de infección bacteriana. An Pediatría. 2001;54(1):69-73.

8. Cha JK, Kwon KH, Byun SJ, Ryoo SR, Lee JH, Chung J, et al. Clinical value of procalcitonin for suspected noso- comial bloodstream infection. Korean J Intern Med. 2017

9. Riedel S, Melendez JH, An AT, Rosenbaum JE, Zenilman JM. Procalcitonin as a marker for the detection of bacteremia and sepsis in the emergency department. Am J Clin Pathol. 2011;135(2):182-9.

10. Sager R, Kutz A, Mueller B, Schuetz P. Procalcitonin-guided diagnosis and antibiotic stewardship revisited. BMC Med . 2017;1-11.

11. Díez-Padrisa N, Bassat Q, Morais L, O'Callaghan-Gordo C, Machevo S, Nhampossa T, et al. Procalcitonin and C-reactive protein as predictors of blood culture positivity among hospitalised children with severe pneumonia in Mozambique. Trop Med Int Heal. 2012;17(9):1100-7.

12. Lanziotti VS et al. Use of biomarkers in pediatric sepsis: literature review. Rev Bras Ter Intensiva. 2016; 28 (4): 472-82.

13. Pontrelli $\mathrm{G}$ et al. Accuracy of serum procalcitonin for the diagnosis of sepsis in neonate and children with systemic inflammatory syndrome: a meta-analysis. BMC Infectious Diseases. 2017; 17: 302.

14. Kim MH, Lim G, Kang SY, Lee WI, Suh JT, Lee HJ. Utility of procalcitonin as an early diagnostic marker of bacteremia in patients with acute fever. Yonsei Med J. 2011;52(2):276-81.

15. Liu HH, Zhang MW, Guo JB, Li J, Su L. Procalcitonin and C-reactive protein in early diagnosis of sepsis caused by either Gram-negative or Gram-positive bacteria. Ir J Med Sci. 2017;186(1):207-12.

16. Charles $\mathrm{PE}$, Ladoire $\mathrm{S}$, Aho $\mathrm{S}$ et al. Serum procalcitonin elevation in critically ill patients at the onset of bacteremia caused by either Gram negative or Gram positive bacteria. BMC Infect Dis. 2008; 8: 38.

17. Watanabe Y; Oikawa N; Hariu M, Fuke R; Seki M. Ability of procalcitonin to diagnose bacterial infection and bacteria types compared with blood culture findings. International Journal of General Medicine. 2016; 9: 325-331.

18. Thomas-Rüddel $D$ et al. Influence of pathogen and focus of infection on procalcitonin values in sepsis patients with bacteremia or candidemia. Critical Care. 2018; 22: 128.

19. Bassetti et al. Comparision between procalcitonin and C-reactive protein to predict blood culture results in ICU patients. Critical Care. 2018; 22.

20. Shuhua Li; Heng Rong; Qinliang G; Yifei C; Zhang G; Jiong Y. Serum procalcitonin levels distinguish Gram-negative bacterial sepsis from Gram-positive bacterial and fungal sepsis. Journal of Research in Medical Sciences. 2016; 21: 39. 
21. Shomali W et al. Can procalcitonin differentiate Staphylococcus aureus from coagulase-negative staphylococci in clustered gram-positive bacteremia?. Diagnostic Microbiology and Infectious Diseases. 2013; 76: 158-61.

22. Martini A, Gottin L, Menestrina N, Schweiger V, Simion D, Vincent J-L: Procalcitonin levels in surgical patients at risk of candidemia. J Infect 2010,60(6):425-430.

23. Brodská H, Maličková K, Adámková V, Benáková H, Stastná MM, Zima T: Significantly higher procalcitonin levels could differentiate Gram-negative sepsis from Gram-positive and fungal sepsis. Clin Exp Med 2012:1-6.

24. Montagna M, Coretti C, Caggiano G: Procalcitonin: a possible marker of invasive fungal infection in high risk patients? J Prev Med Hyg 2011,52(1):38.

25. Cortegiani A, Russotto V, Montalto F, Foresta G, Accurso G, Palmeri C, et al. Procalcitonin as a marker of Candida species detection by blood culture and polymerase chain reaction in septic patients. BMC Anesthesiol. 2014;14(1):9.

26. Miglietta et al. Procalcitonin, C-reactive protein and serum lactacte dehydrogenase in the diagnosis of bacterial sepsis, SIRS and systemic candidiasis. Le infezioni in Medicine. 2015; 3: 230-237.

27. Oussalah et al. Diagnostic accuracy of procalcitonin for predicting blood culture results in patients with suspected bloodstream infection. Medicine. 2015; 94 (44): 1-8

28. Leli C, Ferranti M, Moretti A, et al. Procalcitonin levels in Gram-positive, Gram-negative, and fungal bloodstream infections. Dis Markers. 2015;2015:701480.

29. Charles PE, Ladoire S, Aho S et al (2008) Serum procalcitonin elevation in critically ill patients at the onset of bacteremia caused by either Gram negative or Gram positive bacteria. BMC Infect Dis 8:38

30. Fleischhack G, Cipic D, Juettner J, Hasan C, Bode U. Procalcitonin-a sensitive inflammation marker of febrile episodes in neutropenic children with cancer. Intensive Care Med. 2000;26 Suppl 2:S202-11.

31. Fleischhack G, Kambeck I, Cipic D, Hasan C, Bode U. Procalcitonin in paediatric cancer patients: Its diagnostic relevance is superior to that of C-reactive protein, interleukin 6 , interleukin 8 , soluble interleukin 2 receptor and soluble tumour necrosis factor receptor II. $\mathrm{Br} \mathrm{J}$ Haematol. 2000;111(4):1093-102.

32. Staehler M, Hammer C, Meiser B, Reichart B (1997) Procalcitonin: a new marker for differential diagnosis of acute rejection and bacterial infection in heart transplantation. Transplant Proc 29:584585

33. A1-Nawas B, Shah PM (1996) Procalcitonin in patients with and without immunosuppression and sepsis. Infection 24:434-436

34. Arif T, Phillips RS. Updated systematic review and meta-analysis of the predictive value of serum biomarkers in the assessment and management of fever during neutropenia in children with cancer. Pediatric Blood \& Cancer. 2019; 66 (10): e27887.

\section{Figures}




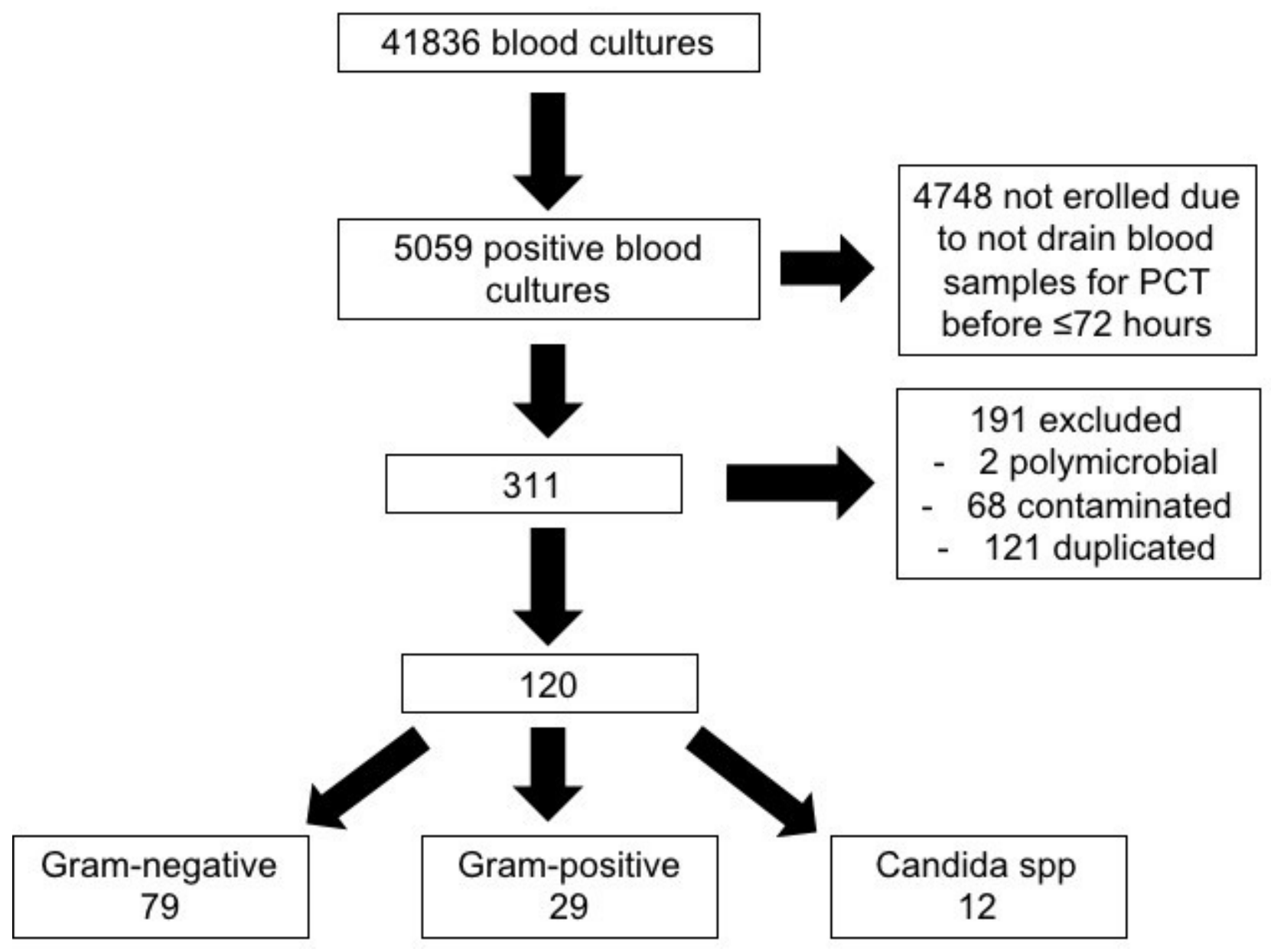

Figure 1

Selection of elegible patients and blood culture samples between January 1, 2011 and April 1,2018 

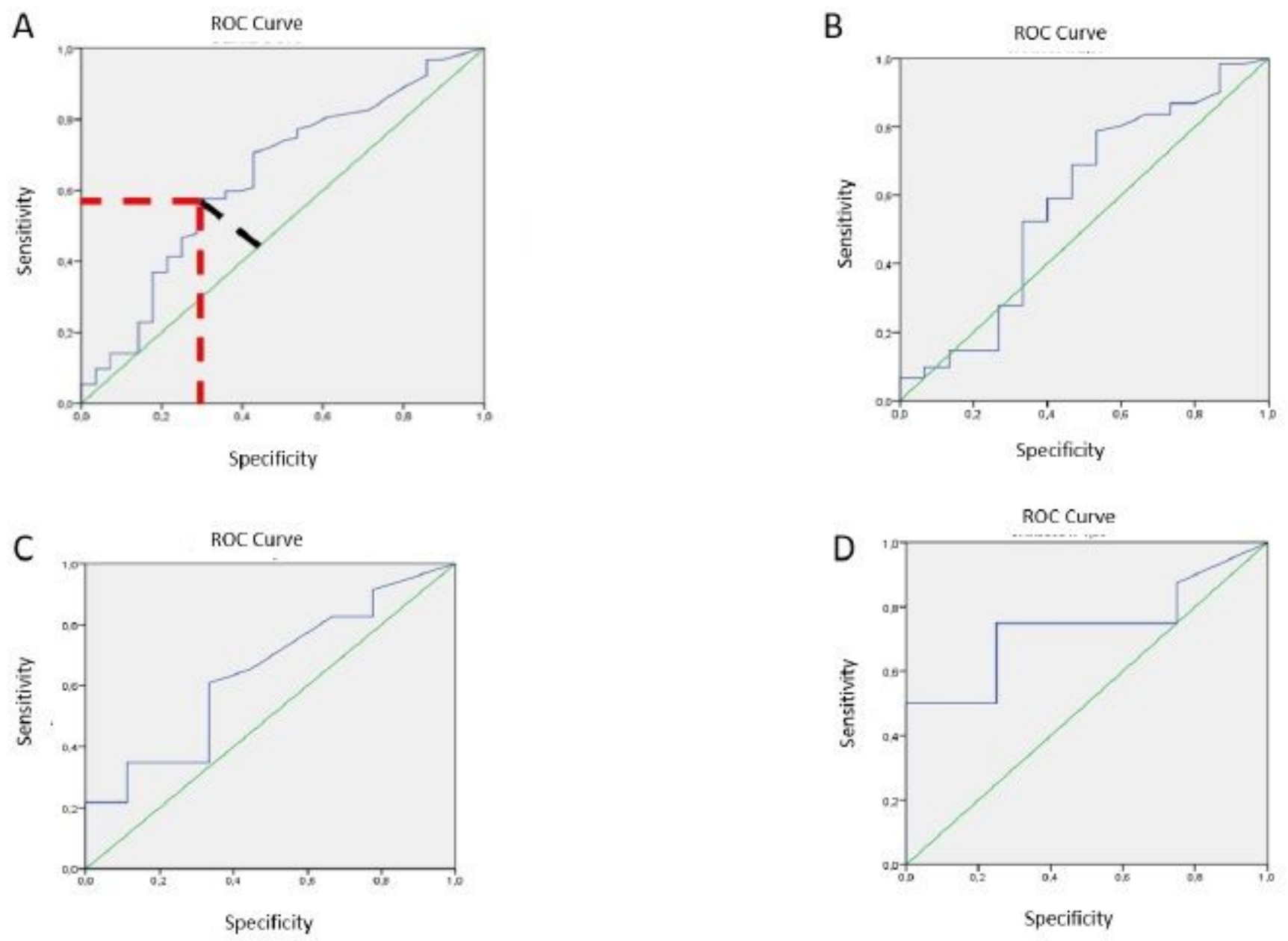

\section{Figure 2}

A) ROC curve of PCT, AUC 0.639 (95\% Cl, 0.519 - 0.760). B) ROC curve of PCT for CGP AUC: 0.581 (95\% $\mathrm{Cl}, 0.402$ - 0.761). C) ROC curve for BGN, AUC: 0.640 (95\% Cl, 0.429 - 0.851). D) ROC curve for molds, AUC: $0.734(95 \% \mathrm{Cl}, 0.444-0.1)$. 